Ida Nuramdhani 1,2, Sheilla Atieno Odhiambo ${ }^{3}$, Carla Hertleer ${ }^{1}$, Gilbert De Mey ${ }^{4}$, Lieva Van Langenhove ${ }^{1}$

${ }^{1}$ Ghent University, Faculty of Engineering and Architecture, Department of Textiles, Technologiepark 907,

9052 Zwijnaarde, Ghent, Belgium

2Department of Textile Chemistry, Polytechnic of Textile Technology, Bandung, Indonesia

3Moi University, Department of Textiles, P.O. BOX 3900, Eldoret 30100, Kenya

${ }^{4}$ Ghent University, Faculty of Engineering and Architecture, Department of Electronics and Information

Systems, Sint-Pietersnieuwstraat 41 B-9000 Ghent, Belgium

\title{
Electric Field Effect on Charge-Discharge Characteristics of Textile-Based Energy Storage Devices: In Search of the Underlying Mechanism
}

\author{
Original Scientific Article \\ Received 03-2016 • Accepted 04-2016
}

\begin{abstract}
The work focused on studying the effect of charging voltage level and the absence of electrode between the measured electrodes on the charge storage profile. The device was applied on a three layered of $5 x$ $5 \mathrm{~cm}$ laminated textile fabric with hydrophobic surface. Stainless steel filament yarns were used as electrodes. As electrolyte, seven layers of the electro-active polymer PEDOT:PSS dispersion was consecutively drop-coated and dried under the temperature of $90-100^{\circ} \mathrm{C}$. During the course of the experiments, the devices were charged and discharged and their response or voltage decay was recorded. The results showed that the higher the charging voltage, the higher the amount of charges stored. The increase in the output voltage was not proportional to the input voltage which means that the device did not behave as either a pure capacitor or a pure battery. The ions movement was confirmed to be involved in the charge-discharge mechanism of the device.
\end{abstract}

Keywords: PEDOT:PSS, energy storage, smart textile, textile capacitor

\section{Introduction}

A generally accepted conception today is that in order to provide people with personalized healthcare, support and information, technological advances should be brought closer to the subject by means of easy-to-use wearable interfaces between devices and humans [1]. This can be achieved through multifunctional fabrics, commonly referred to as electronic textiles (e-textiles) or smart textiles, capable of making daily life healthier, safer and more comfortable. Such fabrics are conceived as innovative and high knowledge-content garments, integrating sensing, actuation, electronic and power functions. An energy supply/storage device is an important component of a smart textile system; it provides the

Corresponding author:

Ida Nuramdhani

Phone: +3292645408

E-mail: ida.nuramdhani@ugent.be required energy to power the system. The successful commercialization of smart garments is hindered by the lack of fully integrated energy storage facilities. There is a need for an embedded device that integrates seamlessly into the textile structure without compromising the comfort and other desirable aspects of the clothing.

A lightweight, flexible, and reliable energy storage device has been developed in our previous study. The reported work in that paper constitutes the fundamental part in the investigation of fabrication of a textile-based energy storage device made with PEDOT:PSS and stainless steel conductive yarns [2]. PEDOT:PSS, an electro-active polymer used in the study, is known to be capable of being an electrode and electrolyte material [3] with high thermal

Tekstilec, 2016, 59(2), 162-167

DOI: 10.14502/Tekstilec2016.59.162-167 
properties, flexibility in terms of its chemical and physical properties as well as a decent electrochemical stability, charge capacity, and ionic conductivity [4]. As an electrode, electro-conductive stainless steel filament yarns showed an encouraging performance. When compared with silver coated PBO (polybenzoxazol), higher capability to store charge with higher voltage value in a longer time was shown by the less expensive stainless steel yarn electrode. In addition, the stainless steel filament yarns are also flexible, and can be comfortably incorporated to textile fabrics. They show good electrical and thermal conductivity with high melting point that give further advantages of using this electro-conductive yarn as a bipolar electrode fabricated textile based energy storage device [2].

From our previous study [5], there is a strong indication that the mechanism involved in the charge storage is mainly through charge separation within the electrolyte and the formation of electric double layer on the electrode surfaces based on the EDLC (electric double layer capacitor) charge storage principle. It is known from our previous results that the accumulated charge in the devices during discharge was proportional to the charging time. Up to a certain extent, the longer the charging time, the more charge was stored in the devices. It was also found that two hours was a sufficient time to charge the cell. However, the charging voltage was not optimized; we used $1.5 \mathrm{~V}$ in most experiments. In this regard, the charging time in relation to charging voltage could have been optimized as well.

The work reported in this paper is focused on studying the effect of charging voltage level on the charge storage profile with a view that it would provide information regarding the possible mechanism of the device. As it was believed that the enhanced conductivity of the device came from the ions transport separated from the conductive PEDOT:PSS, the effect of the absence of electrode between the measured electrodes as given in the standard device (Figure 1) was observed. For this purpose, a modified device (Figure 1), presenting only electrolyte between the two measured electrodes with various distances was prepared. The difference in charging voltage level, the absence of a middle electrode and a distance between electrodes were expected to give results to different level of electric field in the device.

\section{Experimental}

\subsection{Materials}

Two types of energy storage device (so-named device $A$ and $B$ ) as shown in Figure 1 were prepared with different set up of the electrodes. Device A was a reproduction of the standard device that had been developed in our previous work [5], consisting of three strands of stainless steel yarn electrodes with symmetrical distance between the yarns $(1 \mathrm{~mm})$. Device B was a modified version of the device A, where the cell was composed of four strands of electrode yarns with different distances between electrodes, i.e. 1,2 , and $3 \mathrm{~mm}$ respectively.

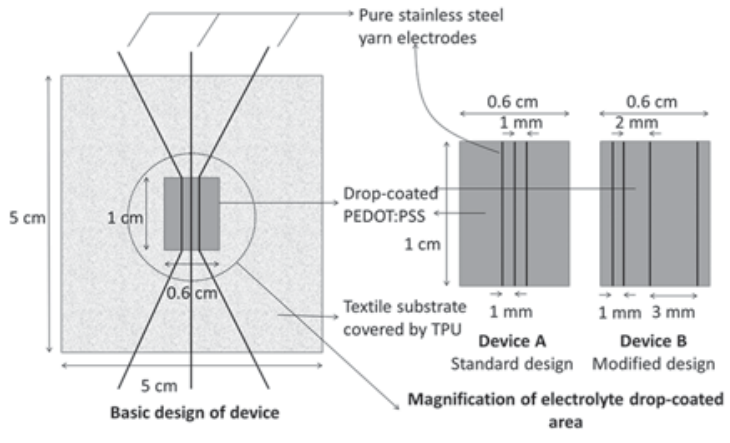

Figure 1: Design of the developed energy storage devices

The cell was assembled on a three layered of $5 \mathrm{~cm} \mathrm{x}$ $5 \mathrm{~cm}$ porous textile fabric laminated together with two pieces of interlining adhesive (hot melt adhesive). Before laminated together, the electrode yarns were stitched to the upper layer of the textile substrate. The surface of the device was then covered with a thermoplastic polyurethane (TPU) layer produced by Epurex Film Company, to give a hydrophobic layer on the fabric surface, except for the area where electrolyte would be applied. The textile substrate used was a twill woven polyester-cotton fabric with a warp density of 42 yarns/cm and a weft density of 29 yarns/cm [6]. The electrode used was pure stainless steel filament yarns from Bekintex, whereas the electro-active polymer PEDOT:PSS (poly $(3,4-$ ethylene dioxythiophene):poly(styrene sulfonate)), dispersion (6\% in water) from OSILLA, was used as the electrolyte. As the content of the electrolyte polymer in the dispersion was low, seven layers of the electrolyte were proportionally drop-coated to the cell using a drop pipette. Each layer of the dropcoated electrolyte was pre-dried in an oven under 
the temperature of $90-100{ }^{\circ} \mathrm{C}$ for 15 minutes before applying the next layers.

\subsection{Method}

In this work, devices A and B were used to study the effect of charging voltage and the presence of electrode between the measured electrodes on the amount of charge accumulated and its charge decay profile. During the course of the experiments, the devices were charged and discharged and their response or voltage decay was recorded. By varying the distance between electrodes $(1 \mathrm{~mm}, 2 \mathrm{~mm}$ and $3 \mathrm{~mm}$ respectively) and the charging voltage level $(1.5 \mathrm{~V}$, $3 \mathrm{~V}, 4.5 \mathrm{~V}$, and $6 \mathrm{~V}$ ) for 300 seconds (short) and 7,200 seconds (long) charging time, the voltage decay was measured using a National Instruments NI PXI 1033, which is a chassis equipped with a voltage generator, a digital voltage meter and a computer interface. The computer was equipped with a developed program that allowed the equipment to charge the developed cells at specified voltages (V) and time (s) and also captured the voltage decay data automatically.

\section{Results}

The graph in Figure 2 shows the profile of voltage decay of the device $\mathrm{A}$ with different charging voltages $(1.5 \mathrm{~V}, 3 \mathrm{~V}, 4.5 \mathrm{~V}$, and $6 \mathrm{~V})$. At each voltage level, the voltage decay was measured on two pairs of electrodes placed at a distance of $1 \mathrm{~mm}$. In general, it can be seen from the graph that the application of higher charging voltage presented higher level of charge that was stored in the device. The decaying rate was also lower when the device was charged with higher charging voltages. Similar results were obtained by measuring the voltage decay of the device A with short (300 seconds) and long $(7,200$ seconds) charge-discharge times.

The graph in Figure 3 shows the profile of voltage decay of the devices A and B shown schematically in Figure 1. The measurements were carried out on the outer electrodes of the device A being a part in a distance of $2 \mathrm{~mm}$ including an intermediate electrode; measurements on device $\mathrm{B}$ were performed on the two electrodes being a part in a distance of $2 \mathrm{~mm}$. For these measurements, the charging voltage was $3 \mathrm{~V}$. In general, it was observed that the presence of electrode between the measured electrodes had an effect on the performance of the device in its ability to store the accumulated charge. The higher amount of accumulated charge was obtained from the device that an electrode was not included between the measured electrodes. This occurred for all measurements with different charging voltages.

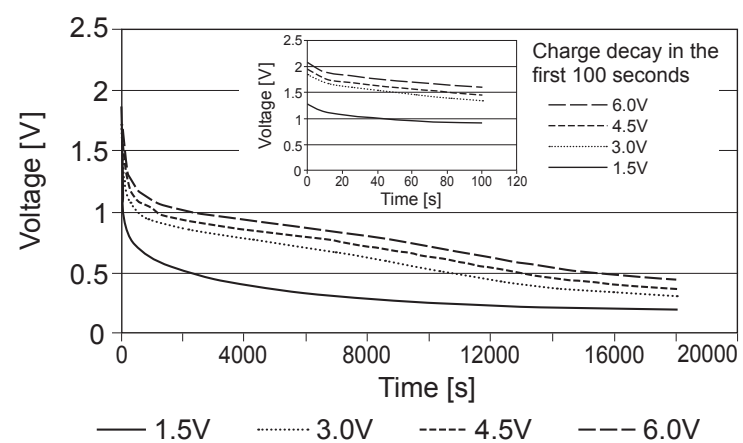

Figure 2: Voltage decay profile of device A with different charging voltages

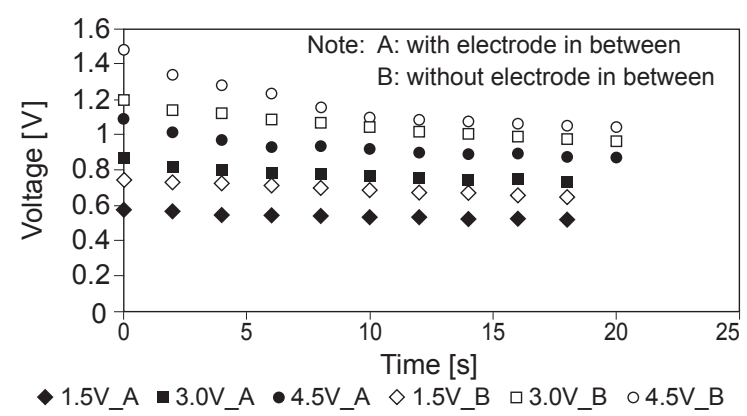

Figure 3: Effect of the presence and absence of an electrode between the measured electrodes to the amount of charge accumulated

\section{Discussion}

The voltage decay profile shown by the graph in Figure 2 explains that the developed device demonstrated its reproducibility in storing charges. The charge storage mechanism was suggested to comprise mainly through ions separation within the PEDOT:PSS electrolyte that then followed by the formation of electrical double layer on the electrode surfaces [2]. The electrode itself, that was a pure stainless steel filament yarn, was known to contain high percentage of chromium atoms with a very thin chrome oxide layer on the yarn surface that made it able to create an insulation layer or dielectric material. The mobile ions transport occurred under the influence of the applied electric field via charging process at particular time and voltage level. It was clear from our previous work [2] that the longer the charging time at a 
constant voltage of $1.5 \mathrm{~V}$, the slower the voltage decay and the higher the charge stored in the devices. There was an indication that slow mechanism involved in the accumulation and storage of charge, that is probably due to the movement of large ions. It was assumed that longer time was needed to drive the large mobile ions formed from the electro-conductive polymer to be fully separated towards each proper end according to their polarity. However, increasing the charging time up to more than 7,200 seconds did not result in significant increase in the amount of accumulated charge.

Likewise, identical charge decay behaviour were also shown by the device given different charging voltage level, i.e. $1.5 \mathrm{~V}, 3.0 \mathrm{~V}, 4.5 \mathrm{~V}$, and $6.0 \mathrm{~V}$ at two different constant charging times: 300 seconds (short) and 7,200 seconds (long) respectively. From the data of long charging times as presented in Figure 2, it was shown that the higher the charging voltage applied during charging time, the more the amount of accumulated charge in the device at its discharge phase. However, the degree of the increase in the stored energy was not proportional to the increase in the charging voltage given. It was observed that just after the power supply stopped, the charge level decayed drastically for each charging level. The data presented in Table 1, show the percentage of voltage decay observed at the starting point ("zero" minute) of discharging phase for each of the different conditions. It would be noted here that the term of "zero minute" (time $=0$ ) is related to the time of initial measurement taken. It would be understood that during charging process, the output voltage remained constant (input voltage = output voltage), until the time before power supply was disconnected. Although the readings were done automatically using the software to minimize errors, there was still a gap between charge-discharge process (just before the zero minute taken) where the voltage could not be recorded.
It can be seen that with a short charging time (300 seconds) the stored charges decayed more from $58 \%$ upwards at each charging voltage. With long charging time (7,200 seconds), the charge decay was less than $40 \%$ for devices constantly charged at $1.5 \mathrm{~V}$ and $3.0 \mathrm{~V}$, while in higher charging voltage values, the decay excided the percentage value of $56 \%$. The data confirmed the typical performance as obtained from the previous result treated with various charging time. Our previous findings [2] have shown that prolonging the charging time did not increase the accumulated charge in the device. This indicates that at certain extent the amount of residual charges stored in the device would not increase significantly. There is an upper limit of the charge density being able to be accumulated in the device.

The charge decay profile also confirmed our previous finding [2] that the stored charges would drastically decrease up to a certain level where the decay was slower and the voltage profile became more constant. In a very long time, the voltage would reach the zero value. From our experiment, it was found that the device could preserve the stored energy with a very slow decrease for 7 days when the voltage reached nearly the zero value. From the data concerning the very slow decay, the graph in Figure 4 shows the relation between charging voltage and detected voltage observed at a given time $(1,000 \mathrm{~s}, 6,000 \mathrm{~s}$ and 10,000 $s$ respectively). In logarithmic scale, the increase of detected voltages at each observed time showed linear relations with consistent slopes number of around 0.1 . From this relation, an empirical model: $V 2=$ $0.35 \ln V 1+C$, was obtained. A constant value of 0.35 was taken from the average constant values of each data series. It can be seen that the data points and the model built based on the empirical data are close enough. Although no physical interpretation can be assumed from this model, the relation between data from each point provided consistent slope and showed a linearity, that were good signs for a

Table 1: Voltage decay observed at the initial measurement (time $=0)$

\begin{tabular}{|c|c|c|c|c|}
\hline \multirow{2}{*}{ Charging [V] } & \multicolumn{2}{|c|}{ With 300 seconds (short) charging } & \multicolumn{2}{c|}{ With 7,200 seconds (long) charging } \\
\cline { 2 - 5 } & Detected voltage [V] & Decay [\%] & Detected voltage [V] & Decay [\%] \\
\hline 1.5 & 0.63 & 58 & 1.267 & 15 \\
\hline 3.0 & 0.96 & 68 & 1.832 & 39 \\
\hline 4.5 & 1.16 & 74 & 1.981 & 56 \\
\hline 6.0 & 1.34 & 78 & 2.063 & 65 \\
\hline
\end{tabular}


consistent data series observed. At the same time, it was also shown that the charging voltage (input) was not related proportionally to the detected voltage (output) at several particular times. The doubling of the charging voltage did not result in a doubled detected voltage. That indicated that the device did not act as a pure capacitor because in capacitor, the increase in input voltage is proportionally increasing the output voltage. The device did not act as a pure battery either because a constant output voltage was expected in battery, regardless the input voltage level given to the device. However, the functional behavior of the device is still questionable up to this point.

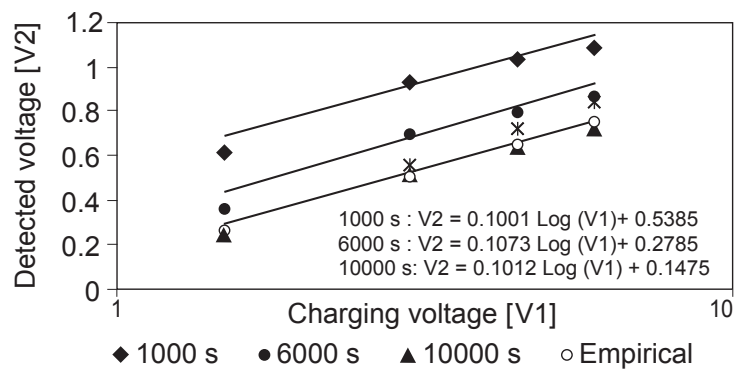

Figure 4: Log scale relations between charging voltage and detected voltage

As mentioned above, the capacitive ability of the electro-active polymer PEDOT:PSS is associated with transport of charge carriers to the interface between the polymer and the yarn electrodes. Some characterization studies using SEM-EDX imaging and FTIR spectroscopy found the evidence of ions transport and redox reversibility in PEDOT:PSS $[7,8]$. The ability to transport charges itself is caused by the presence of overlapping $\mathrm{C}=\mathrm{C}$ bonds that give $\pi$ orbitals along the polymer materials. They become electrically conductive due to mobile carriers (holes) created in the oxidized state [9]. From our previous work [5], it was suggested that dielectric polarization of PEDOT:PSS stimulated by the electric field applied through the charging process was the reason for charge storage capability of our developed device. As the stainless steel electrode yarn was not chemically inert and covered by very thin layer of chrome oxide $\left(\mathrm{Cr}_{2} \mathrm{O}_{3}\right)$ that can prevent further oxidation of the steel, it became capable of creating an insulating layer interface between the metal and the polymer $[10,11]$. It has been reported that PEDOT:PSS film has no crystalline structure, it presents a strong effect on the charge transport, and therefore, the conductivity of the polymer was assumed to be due to its disordered conjugated system [12]. When the charging process applied, an electric double layer was observed in both electrodes. Charge recombination then occurred just after the charging process stopped, when the separated ions from the polymer got back to their initial random arrangement $[2,5]$. The graph shown in Figure 3 confirmed this assumption.

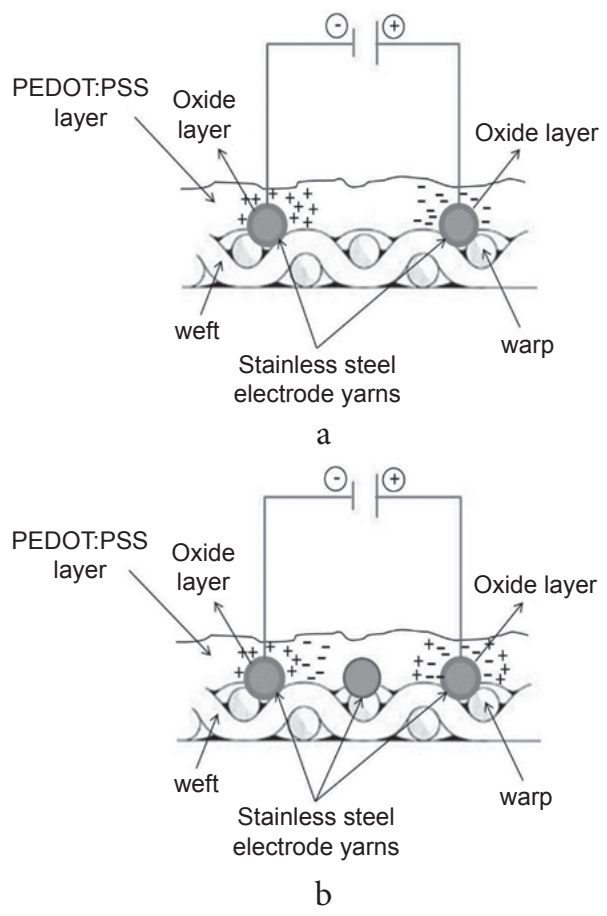

Figure 5: Cross-section visualisation of the device cell: (a) no middle electrode, (b) with middle electrode

From the graph in Figure 3 it can be seen that the presence of the intermediate electrode affected the amount of charges accumulated in the device. This could be because the ions transport towards or from the end electrodes (anode or cathode) in the cell was restricted by the presence of the electrode between the measured electrodes (or the intermediate electrode). As it can be seen in the cross-section visualization of the cell presented in Figure 5, unlike electrons that can undergo a chemical reaction with metal, ions cannot. Therefore, the moving ions cannot penetrate and even their transport was restricted by the intermediate metal electrode. As the charge transport occurred on the surface of the device, the transport pathway also became longer as the ions would pass the surface of the intermediate metal electrode. These results proved further that the ionic transport principle occurred in the charge-discharge 
process of the device. This also indicates that ions cannot penetrate the metal electrode.

\section{Conclusion}

The results obtained from this work shows that the strength of electric field applied via charging process affect the capability of the device to store energy. The higher the charging voltage, the higher the amount of accumulated charge stored. However, the increase in the amount of charge stored is not proportional to the increase of the charging voltage applied. This leads to the assumption that the device does operate neither as a pure capacitor nor a pure battery.

The results obtained with the presence of an intermediate third electrode proves that ion movement is the basic mechanism. Ions cannot penetrate into a metallic electrode so that this third electrode inhibits the flow of ions.

The result also proved the ions movement principle involved in the charge-discharge mechanism of the device. Further experiments and characterization are needed to give a conclusive understanding about the charge storage mechanism of the device.

\section{References}

1. SCHWARZ, Anne, Van LANGENHOVE, Lieva, GUERMONPREZ, Philippe, DEGUILLEMOMT, Denis. A roadmap on smart textiles. TextileProgress, 2010, 42(2), 99-180, doi: 10.1080/ 00405160903465220.

2. ODHIAMBO, Sheilla Atieno, De MEY, Gilbert, HERTLEER, Carla, SCHWARZ, Anne, Van LANGENHOVE, Lieva. Discharge characteristics of poly(3,4-ethylene dioxythiophene): poly (styrenesulfonate) (PEDOT:PSS) textile batteries; comparison of silver coated yarn electrode devices and pure stainless steel filament yarn electrode devices. Textile Research Journal, 2013, 84(4), 347-354, doi: 10.1177/0040517513481871.

3. BHATACARYA, Rabin, de KOK, Margareet M., ZHOU, J. Rechargeable electronic textile battery. Applied Physiscs Letters, 2009, 95(22), 223305-1-1223305-3, doi: 10.1063/1.3269907.

4. CRISPIN, X., JAKOBSSON, F. L. E., CRISPIN, A., GRIM, P. C. M., ANDERSON, P., VOLODIN, A., van HAESENDONCK, C., Van der AUWER-
AER, M., SALANECK, W. R., BERGGREN, M. The origin of the high conductivity of poly $(3,4-$ ethylenedioxythiophene)-poly(styrenesulfonate) (PEDOT-PSS) plastic electrodes. Chemistry of Materials, 2006, 18(18), 4354-4360, doi: 10.1021/ cm061032+.

5. ODHIAMBO, Sheilla Atieno, De MEY, Gilbert, HERTLEER, Carla, Van LANGENHOVE, Lieva. Reliability testing of PEDOT:PSS capacitors integrated into textile fabrics. Maintenance and Reliability Journal, 2014. 3, 440-445.

6. KAZANI, Ilda. Study of screen-printed electroconductive textile materials : thesis. Ghent: Ghent University, 2012.

7. OUYANG, Jianyong, XU, Qianfei, CHU, ChiWei, YANG, Yang, LI, Gang, SHINAR, Joseph. On the mechanism of conductivity ehancement in poly (3,4-ethylenedioxythiopene):poly(styren esulfonate) film through solvent treatment. Polymer, 2004, 45(25), 8443-8450, doi: 10.1016/ j. polymer.2004.10.001.

8. COLLAZOS-CASTRO, Jorge E., POLO, José L., HERNÁNDEZ-LABRADO, Gabriel R., PADIAL-CAÑETE, Vanesa, GARCÍA-RAMA, Concepción. Bioelectrochemical control of neural cell development on conducting polymers. Biomaterials, 2010, 31(35), 9244-9255, doi: 10.1016/ j.biomaterials.2010.08.057.

9. ELSCHNER, Andreas, KIRCHMEYER, Stephan, LOVENICH, Wilfried, MERKER, Udo, REUTER, Knud. Properties of PEDOT:PSS. In: PEDOT: principles and applications of intrinsically conductive polymer. New York : CRC Press, 2011, pp. 123-148.

10. SUNDFORS, Fredrik, GUSTAFSSON, Henrik, IVASKA, Ari, KVARNSTRÖM, Carita Characterisation of the aluminium-electropolymerised poly(3,4-ethylenedioxythiopene) system. Journal of Solid State Electrochemistry, 2010, 14(7), 1185-1195.

11. INGANÄS, O., Lundström, I. Electronic properties of metal/polypyrrole junctions. Synthetic Metals, 1984, 10(1), 5-12, doi: 0.1016/0379-6779 (84)90074-2.

12. ELSCHNER, Andreas, KIRCHMEYER, Stephan, LOVENICH, Wilfried, MERKER, Udo, REUTER, Knud. Properties of PEDOT:PSS. In: PEDOT: principles and applications of intrinsically conductive polymer. New York : CRC Press, 2011, pp. 21-29. 